\title{
On a class of generalized stochastic Browder mixed variational inequalities
}

\author{
Chao Min ${ }^{1,2^{*}} \mathbb{D}$, Fei-fei Fan' ${ }^{1}$ Zhao-zhong Yang ${ }^{3}$ and Xiao-gang Li ${ }^{3}$
}

"Correspondence:

minchao@swpu.edu.cn

'School of Science, Southwest

Petroleum University, Chengdu,

P.R. China

${ }^{2}$ Institute for Artificial Intelligence,

Southwest Petroleum University,

Chengdu, P.R. China

Full list of author information is

available at the end of the article

\section{Springer}

\begin{abstract}
In this paper, we introduce a class of stochastic variational inequalities generated from the Browder variational inequalities. First, the existence of solutions for these generalized stochastic Browder mixed variational inequalities (GS-BMVI) are investigated based on FKKM theorem and Aummann's measurable selection theorem. Then the uniqueness of solution for GS-BMVI is proved based on strengthening conditions of monotonicity and convexity, the compactness and convexity of the solution sets are discussed by Minty's technique. The results of this paper can provide a foundation for further research of a class of stochastic evolutionary problems driven by GS-BMVI.
\end{abstract}

Keywords: GS-BMVI; Gwinner's section theorem; Aummann's measurable selection; Minty's technique; Solution sets

\section{Introduction}

In the deterministic cases, there have been a lot of research to describe the properties of different types of variational inequalities [1-4], including the sufficient conditions of the existence and the uniqueness of the solutions, and the compactness and connectivity of the solution sets. Stochastic variational inequalities are generalized from variational inequalities due to the uncertainties which are often involved in practical problems. Stochastic variational inequalities are a powerful tool for the study of various stochastic problems and stochastic control problems [5-7]. The research on stochastic variational inequalities can not only solve practical optimization problems in the fields of economics, traffic networks and engineering, but also play an important role in promoting the research of stochastic functional analysis theory and applications. In recent years, there has been a lot of work focusing on stochastic variational inequalities. Existence and continuity results are proved for stochastic weighted variational inequalities in a non-pivot Hilbert space in [8]. The solvability, differentiability, and convexity of the two-stage stochastic programming and the convergence of the sample average approximation for a two-stage stochastic variational inequality are established in [9]. The progressive hedging algorithm is studied for multistage stochastic variational inequality problems under monotonicity and the stochastic complementarity problems are explored numerically in a linear two-stage formulation in [10]. Ren et al. [11] established various limit theorems for one-dimensional

(c) The Author(s) 2020. This article is licensed under a Creative Commons Attribution 4.0 International License, which permits use sharing, adaptation, distribution and reproduction in any medium or format, as long as you give appropriate credit to the original author(s) and the source, provide a link to the Creative Commons licence, and indicate if changes were made. The images or other third party material in this article are included in the article's Creative Commons licence, unless indicated otherwise in a credit line to the material. If material is not included in the article's Creative Commons licence and your intended use is not permitted by statutory regulation or exceeds the permitted use, you will need to obtain permission directly from the copyright holder. To view a copy of this licence, visit http://creativecommons.org/licenses/by/4.0/. 
stochastic variational inequalities with Yamada-Watanabe-type conditions on the coefficients. Besides, the stochastic variational inequalities are close to the stochastic complementarity problems, and for the theoretical research of the stochastic complementarity problems, the relevant properties of their solutions are usually discussed. For example, Zhang and Huang [12] studied a class of stochastic generalized set-valued quasicomplementarity problems and proved the existence of their solutions and the convergence of random sequences generated by a new random iterative algorithm. In [13], the authors showed that the requirements of solvability for complementarity problems over cones are different and that some analogous verifiable integration-free statement can be proved for stochastic complementarity problems.

In this paper, we will study a new class of stochastic variational inequalities generated from the Browder variational inequalities: Let $(\Omega, \mathcal{F})$ be a measurable space and $E$ be a Banach space with a nonempty subset $K$; let $E^{*}$ be the dual space of $E$. We formulate a class of generalized stochastic Browder mixed variational inequality in this paper as follows:

$$
\langle Q(\omega, u), v-u\rangle+\phi(\omega, v)-\phi(\omega, u) \geq 0
$$

where $Q: \Omega \times K \rightarrow E^{*}$ denotes a stochastic mapping, $\phi: \Omega \times K \rightarrow(-\infty,+\infty]$ represents a stochastic functional and $\phi \not \equiv+\infty,\langle\cdot, \cdot\rangle$ denotes the duality pairing of $E^{*}$ and $E$.

Our main work considers the above GS-BMVI (1) by finding a measurable selection $\xi: \Omega \rightarrow K$ such that

$$
\langle Q(\omega, \xi(\omega)), v-\xi(\omega)\rangle+\phi(\omega, v)-\phi(\omega, \xi(\omega)) \geq 0
$$

for $\forall v \in K$, and discusses the relevant properties of its solutions.

The paper is structured as follows. First, some essential basic definitions and preliminary facts needed in the sequel are introduced in Sect. 2. Then the existence, uniqueness, compactness, and convexity of solution sets for GS-BMVI (1) are discussed and proved in Sect. 3. At last, the core work and methods of this paper are summarized and further work is also described in Sect. 4.

\section{Preliminaries}

In this section we recall some prerequisites and assumptions regarding set-valued mappings and a fixed point theorem that will be used later on.

Let $E$ be a Hausdorff topological vector space and $(\Omega, \mathcal{F})$ be a measurable space; $\mathcal{B}(E)$ represents the $\sigma$-algebra of all Borel subsets of $E, C B(E)$ denotes the family of all nonempty closed sets of $E$, and $\mathcal{F} \times \mathcal{B}(E)$ is the family of all measurable sets in $\Omega \times E$.

Definition 2.1 A mapping $G: \Omega \rightarrow 2^{E}$ is $(\mathcal{F}, \mathcal{B}(E))$-measurable, if for any open set $B \subseteq$ $\mathcal{B}(E)$,

$$
G^{-1}(B)=\{\omega \in \Omega, \Gamma(\omega) \cap B \neq \emptyset\} \in \mathcal{F} .
$$

Definition 2.2 A mapping $G: \Omega \times E \rightarrow 2^{E}$ is measurable, if $G(\cdot, v): \Omega \rightarrow 2^{E}$ is measurable for any $v \in E$. 
Definition 2.3 A mapping $\xi: \Omega \rightarrow E$ is a stochastic fixed point of a measurable mapping $G: \Omega \times E \rightarrow 2^{E}$, if $\xi$ is measurable and $\xi(\omega) \in G(\omega, \xi(\omega))$.

Definition 2.4 ([14]) Let $K$ be a nonempty subset of a Hausdorff topological space $E$ and $G: K \rightarrow 2^{E}$ be a set-valued mapping. If for any finite set $\left\{k_{1}, k_{2}, \ldots, k_{n}\right\} \subset K$, there exists a finite subset $\left\{y_{1}, y_{2}, \ldots, y_{n}\right\} \subset E$ such that for any subset $I \subset\{1, \ldots, n\}$,

$$
\operatorname{co}\left\{y_{i}: i \in I\right\} \subset \bigcup_{i \in I} G\left(k_{i}\right)
$$

then $G$ is a generalized KKM mapping. In particular, when $K=E$ and $k_{i} \equiv y_{i}(i=1, \ldots, n)$, then $G$ is a KKM mapping.

Theorem 2.1 (FKKM theorem, [15]) Let $K$ be a nonempty subset of a Hausdorff topological space E. Suppose that a KKM mapping $G: K \rightarrow 2^{E}$ is closed for each $k \in K$, and $G\left(k_{0}\right)$ is compact for $k_{0} \in K$, then

$$
\bigcap_{k \in K} G(k) \neq \emptyset .
$$

Definition 2.5 ([16]) A Hausdorff topological space $E$ is: $(i)$ a Polish space if $E$ is separable and metrizable by a complete metric; and $(i i)$ a Suslin space if $E$ is a Hausdorff topological space and a continuous image of a Polish space.

Notice that a separable Banach space is a Suslin space.

Lemma 2.1 (Aumann's measurable selection, [17]) For a Hausdorff topological space $(\Omega, \mathcal{F}, P)$, and a separable Hilbert space $E$, if there exists a measurable mapping $\Gamma: \Omega \rightarrow$ $2^{E}$ such that

$$
\operatorname{graph}(\Gamma)=\{(\omega, x) \in \Omega \times E: x \in \Gamma(\omega)\} \in \mathcal{F} \times \mathcal{B}(E)
$$

then $\Gamma(\cdot)$ is measurable, and $\Gamma$ has a measurable selection $\xi: \Omega \rightarrow K$ such that $\xi(\omega) \in$ $\Gamma(\omega)$ is valid for all $\omega \in \Omega$.

\section{Main results}

In this section, we mainly discuss the properties of solution sets for GS-BMVI (1).

Theorem 3.1 Let E be a separable Banach space with a nonempty closed and convex subset $K$, and let $E^{*}$ be the dual space of E. Suppose that the stochastic functional $\phi: \Omega \times K \rightarrow(-\infty,+\infty](\phi \not \equiv+\infty)$ is convex and lower semicontinuous, while the stochastic mapping $Q: \Omega \times K \rightarrow E^{*}$ is continuous. If the following conditions are satisfied:

(i) The mapping $G: \Omega \times K \rightarrow 2^{K}$ satisfies

$$
G(\omega, v)=\{u \in K:\langle Q(\omega, u), v-u\rangle+\phi(\omega, v)-\phi(\omega, u) \geq 0\} .
$$

(ii) There exists a compact subset $K^{\prime} \subset E$ and $k \in K \cap K^{\prime}$ such that

$$
\langle Q(\omega, u), k-u\rangle+\phi(\omega, k)-\phi(\omega, u)<0
$$

for $\forall u \in K \backslash K^{\prime}$. 
(iii) $\langle Q(\omega, u), v-u\rangle+\phi(\omega, v)$ is quasiconvex and upper semicontinuous about $v \in K$.

(iv) $X$ is a finite-dimensional subspace of E. If for any finite-dimensional section $D=K \cap X$ and any net $\left\{u_{\alpha}\right\} \subset K \cap K^{\prime}$ with $\left\{u_{\alpha}\right\} \rightarrow u \in D$, one has

$$
\left\langle Q\left(\omega, u_{\alpha}\right), v-u_{\alpha}\right\rangle+\phi(\omega, v) \geq \phi\left(\omega, u_{\alpha}\right)
$$

then it follows that

$$
\langle Q(\omega, u), v-u\rangle+\phi(\omega, v) \geq \phi(\omega, u)
$$

for $\forall v \in D$.

(v) $\phi(\omega, u)-\langle Q(\omega, u), v-u\rangle$ is lower semicontinuous about $u \in K$.

Then GS-BMVI (1) has a solution.

Proof We aim here at proving the existence of solutions of GS-BMVI (1). The proof is divided into three parts:

1. We will prove that (a) $G$ has a measurable image; (b) $G$ is a KKM mapping; and (c) $\bigcap_{v \in K} G(\omega, v) \neq \emptyset$ a.s. $\omega \in \Omega$.

(a) Let $B$ be an open subset of $K$. Since $E$ is a separable Banach space, we suppose that there exists a sequence $\left\{v_{n}\right\}$ in $K$. Then for each $u \in K$, we have

$$
\begin{aligned}
G^{-1}(B) & =\{(\omega, u) \in \Omega \times K: G(\omega, v) \cap B \neq \emptyset\} \\
& =\bigcap\left\{(\omega, u) \in \Omega \times K: G\left(\omega, v_{n}\right) \cap B \neq \emptyset\right\} \\
& =\left\{(\omega, u) \in \Omega \times K: \bigcap_{u \in B} G\left(\omega, v_{n}\right) \neq \emptyset\right\} \in \mathcal{F}
\end{aligned}
$$

and $G$ is measurable. According to Theorem 3.5 in [18], $\operatorname{Gr}(G)$ is $\mathcal{F} \times \mathcal{B}$-measurable, thus $G$ has a measurable image.

(b) Arguing by contradiction that $G$ is not a KKM mapping, there exists a finite set $\left\{v_{1}, v_{2}, \ldots, v_{n}\right\} \subset K$ such that

$$
\operatorname{co}\left(v_{1}, v_{2}, \ldots, v_{n}\right) \not \subset \bigcup_{i=1}^{n} G\left(\omega, v_{i}\right)
$$

so $\bar{v} \in \operatorname{co}\left(v_{1}, v_{2}, \ldots, v_{n}\right), \bar{v}=\sum_{i=1}^{n} \lambda_{i} v_{i}\left(\lambda_{i} \geq 0, \sum_{i=1}^{n} \lambda_{i}=1, i=1,2, \ldots, n\right)$, and $\bar{v} \notin \bigcup_{i=1}^{n} G(\omega$, $\left.v_{i}\right)$, thus $\bar{v} \notin G\left(\omega, v_{i}\right)$, that is,

$$
\left\langle Q(\omega, \bar{v}), v_{i}-\bar{v}\right\rangle+\phi\left(\omega, v_{i}\right)-\phi(\omega, \bar{v})<0
$$

and $\phi\left(\omega, v_{i}\right)<+\infty, i=1,2, \ldots, n$.

According to condition (iii), $\langle Q(\omega, u), v-u\rangle+\phi(\omega, v)$ is quasiconvex, so

$$
\{v \in K:\langle Q(\omega, \bar{v}), v-\bar{v}\rangle+\phi(\omega, v)-\phi(\omega, \bar{v})<0\}
$$

is a convex set. Then (4) and (5) lead to

$$
\bar{v}=\sum_{i=1}^{n} \lambda_{i} v_{i} \in\{v \in K:\langle Q(\omega, \bar{v}), v-\bar{v}\rangle+\phi(\omega, v)-\phi(\omega, \bar{v})<0\}
$$


SO

$$
\langle Q(\omega, \bar{v}), \bar{v}-\bar{v}\rangle+\phi(\omega, \bar{v})-\phi(\omega, \bar{v})<0
$$

while

$$
\langle Q(\omega, \bar{v}), \bar{v}-\bar{v}\rangle+\phi(\omega, \bar{v})-\phi(\omega, \bar{v})=0
$$

which yields a contradiction; therefore $G$ is a KKM mapping.

(c) The set $G(\omega, v)$ is weakly closed in $E$ for all $v \in K$. Indeed, taking a sequence $\left\{u_{\alpha}\right\} \subset$ $G(\omega, v)$ with $\left\{u_{\alpha}\right\} \rightarrow u$, one has

$$
\left\langle Q(\omega, v), v-u_{\alpha}\right\rangle+\phi(\omega, v)-\phi\left(\omega, u_{\alpha}\right) \geq 0
$$

that is,

$$
\left\langle Q(\omega, v), v-u_{\alpha}\right\rangle+\phi(\omega, v) \geq \phi\left(\omega, u_{\alpha}\right)
$$

On the basis of conditions $(i v)$ and $(v)$, we get

$$
\langle Q(\omega, v), v-u\rangle+\phi(\omega, v) \geq \phi(\omega, u)
$$

so

$$
\langle Q(\omega, v), v-u\rangle+\phi(\omega, v)-\phi(\omega, u) \geq 0
$$

which means that $u \in G(\omega, v)$.

It can be known that there must exists $u \notin G(\omega, v)$ for all $u \in K \backslash K^{\prime}$, due to condition (ii). Indeed, if, on the contrary, there exists $u$ such that $u \in G(\omega, v)$, then $u \in K$ and $u \in$ $K^{\prime}$, so $G(\omega, v) \subset K^{\prime}$ due to $G(\omega, v) \subset K$, therefore $G(\omega, v)$ is compact. From Theorem 2.1, $\bigcap_{v \in K} G(\omega, v) \neq \emptyset$.

2. Take a mapping $\Gamma: \Omega \rightarrow 2^{K}$ such that $\Gamma(\omega)=\bigcap_{v \in K} G(\omega, v)$. Suppose that $\left\{v_{i}\right\}_{i=1}^{\infty}$ is a dense subset in $K$. It should be proved that

$$
\bigcap_{v \in K} G(\omega, v)=\bigcap_{i=1}^{\infty} G\left(\omega, v_{i}\right)
$$

Obviously, $\bigcap_{v \in K} G(\omega, v) \subset \bigcap_{i=1}^{\infty} G\left(\omega, v_{i}\right)$, so we just need to get $\bigcap_{i=1}^{\infty} G\left(\omega, v_{i}\right) \subset$ $\bigcap_{v \in K} G(\omega, v)$.

Arguing by the contradiction that $\bigcap_{i=1}^{\infty} G\left(\omega, v_{i}\right) \not \subset \bigcap_{v \in K} G(\omega, v)$, so $\exists u_{0} \in \bigcap_{i=1}^{\infty} G\left(\omega, v_{i}\right)$ and $u_{0} \notin \bigcap_{v \in K} G(\omega, v)$, it follows that $\exists v_{0} \in K$ such that $u_{0} \notin G\left(\omega, v_{0}\right)$, that is,

$$
\left\langle Q\left(\omega, u_{0}\right), v_{0}-u_{0}\right\rangle+\phi\left(\omega, v_{0}\right)-\phi\left(\omega, u_{0}\right)<0
$$

There exists $\left\{v_{n_{j}}\right\} \subset\left\{v_{i}\right\}$ such that $\left\{v_{n_{j}}\right\} \rightarrow\left\{v_{0}\right\}$ because $\left\{v_{i}\right\}_{i=1}^{\infty}$ is a countable dense subset of $K$. And since

$$
u_{0} \in \bigcap_{i=1}^{\infty} G\left(\omega, v_{i}\right) \subset \bigcap_{j=1}^{\infty} G\left(\omega, v_{n_{j}}\right)
$$


we have

$$
\left\langle Q\left(\omega, u_{0}\right), v_{n_{j}}-u_{0}\right\rangle+\phi\left(\omega, v_{n_{j}}\right)-\phi\left(\omega, u_{0}\right) \geq 0
$$

for $\forall j \geq 1$.

Since $\langle Q(\omega, u), v-u\rangle+\phi(\omega, v)$ is upper semicontinuous by condition (iii), then

$$
\begin{aligned}
\left\langle Q\left(\omega, u_{0}\right), v_{0}-u_{0}\right\rangle+\phi\left(\omega, v_{0}\right) & \geq \varlimsup_{j \rightarrow \infty}\left\{\left\langle Q\left(\omega, u_{0}\right), v_{n_{j}}-u_{0}\right\rangle+\phi\left(\omega, v_{n_{j}}\right)\right\} \\
& \geq \phi\left(\omega, u_{0}\right),
\end{aligned}
$$

that is,

$$
\left\langle Q\left(\omega, u_{0}\right), v_{0}-u_{0}\right\rangle+\phi\left(\omega, v_{0}\right)-\phi\left(\omega, u_{0}\right) \geq 0
$$

from which we obtain a contradiction $u_{0} \notin G\left(\omega, v_{0}\right)$, so

$$
\bigcap_{i=1}^{\infty} G\left(\omega, v_{i}\right) \subset \bigcap_{v \in K} G(\omega, v)
$$

thus

$$
\bigcap_{v \in K} G(\omega, v)=\bigcap_{i=1}^{\infty} G\left(\omega, v_{i}\right) .
$$

3. Consider a mapping $\Gamma: \Omega \rightarrow 2^{K}$ such that $\Gamma(\omega)=\bigcap_{v \in K} G(\omega, v)$. It follows that

$$
\begin{aligned}
\operatorname{graph}(\Gamma) & =\left\{(\omega, u): u \in \Gamma(\omega)=\bigcap_{i=1}^{\infty} G\left(\omega, v_{i}\right)\right\} \\
& =\bigcap_{i=1}^{\infty}\left\{(\omega, u): u \in G\left(\omega, v_{i}\right)\right\} \in \mathcal{F} \times \mathcal{B}(K) .
\end{aligned}
$$

From Lemma 2.1, a measurable selection $\xi: \Omega \rightarrow K$ can be found such that $\xi(\omega) \in$ $\bigcap_{v \in K} G(\omega, v)$, so GS-BMVI (1) has a solution.

Remark 3.1 Theorem 3.1 is generalized of Browder variational inequality for a stochastic mapping $Q: \Omega \times K \rightarrow E^{*}$ and stochastic functional $\phi: \Omega \times K \rightarrow(-\infty,+\infty]$, which is an extension of the results in Chuong and Thuan [19] and Wu [20]. The existence theorem for many other types of stochastic variational inequalities can be obtained from Theorem 3.1.

Corollary 3.1 Let E be a separable Banach space with a nonempty compact and convex subset $K$, and let $E^{*}$ be the dual space of $E$. Suppose that a stochastic functional $\phi: \Omega \times$ $K \rightarrow(-\infty,+\infty](\phi \not \equiv+\infty)$ is convex and lower semicontinuous, while a stochastic mapping $Q: \Omega \times K \rightarrow E^{*}$ is continuous. If the following conditions are satisfied:

(i) The mapping $G: \Omega \times K \rightarrow 2^{K}$ satisfies

$$
G(\omega, v)=\{u \in K:\langle Q(\omega, u), v-u\rangle+\phi(\omega, v)-\phi(\omega, u) \geq 0\},
$$


(ii) $\langle Q(\omega, u), v-u\rangle+\phi(\omega, v)$ is quasiconvex and upper semicontinuous about $v \in K$.

(iii) $\phi(\omega, u)-\langle Q(\omega, u), v-u\rangle$ is lower semicontinuous about $u \in K$.

Then GS-BMVI (1) has a solution.

Proof Take $K^{\prime}=K$, so $K \backslash K^{\prime}$ because $K$ is compact, then there exists $k \in K$ such that $G(\omega, k)$ is compact, for which $\bigcap_{v \in K} G(\omega, v) \neq \emptyset$.

We need to prove that condition (iv) in Theorem 3.1 is valid in this corollary. Indeed, for any $v \in D \subset K$ and any net $\left\{u_{a}\right\} \rightarrow u \in D$, if

$$
\phi\left(\omega, u_{\alpha}\right)-\left\langle Q\left(\omega, u_{\alpha}\right), v-u_{\alpha}\right\rangle \leq \phi(\omega, v)
$$

for $\forall v \in D$, there holds

$$
\begin{aligned}
\phi(\omega, u)-\langle Q(\omega, u), v-u\rangle & \leq \frac{\lim }{\alpha}\left(\phi\left(\omega, u_{\alpha}\right)-\left\langle Q\left(\omega, u_{\alpha}\right), v-u_{\alpha}\right\rangle\right) \\
& \leq \phi(\omega, v)
\end{aligned}
$$

which implies that condition (iv) of Theorem 3.1 holds. Therefore, the solution of GSBMVI (1) exists.

Corollary 3.2 Let E be a reflexive Banach space with a bounded closed and convex subset $K$, and let $E^{*}$ be the dual space of $E$. Suppose that a stochastic functional $\phi: \Omega \times K \rightarrow$ $(-\infty,+\infty](\phi \not \equiv+\infty)$ is convex and lower semicontinuous, while a stochastic mapping $Q$ : $\Omega \times K \rightarrow E^{*}$ is continuous. If the following conditions are satisfied:

(i) The mapping $G: \Omega \times K \rightarrow 2^{K}$ satisfies

$$
G(\omega, v)=\{u \in K:\langle Q(\omega, u), v-u\rangle+\phi(\omega, v)-\phi(\omega, u) \geq 0\},
$$

as in the previous theorem, $G$ has a measurable graph.

(ii) $\langle Q(\omega, u), v-u\rangle+\phi(\omega, v)$ is quasiconvex and weakly upper semicontinuous about $v \in$ $K$.

(iii) $\phi(\omega, u)-\langle Q(\omega, u), v-u\rangle$ is weakly lower semicontinuous about $u \in K$.

Then GS-BMVI (1) has a solution.

Proof It's easy to see that $K$ is bounded, closed, and convex because it is weakly compact and convex in the reflexive Banach space $E$. Moreover, strongly convergent and weakly convergent are equivalent notions in a finite-dimensional space, so lower(upper) semicontinuous and weakly lower(upper) semicontinuous are equivalent notions in a finitedimensional space, too. For the bounded, closed, and convex subset $K$ in the reflexive Banach space E, if we weaken the conditions which are presented in conditions (ii) and (iii), the existence of solution for GS-BMVI (1) could be reached.

Example 3.1 Let $E=R^{2}$ and $K=[0,+\infty) \times[0,+\infty)$. Suppose that $\omega$ comes from a normal distribution. Define $\phi: \Omega \times K \rightarrow(-\infty,+\infty]$ by

$$
\phi(\omega, v):=\omega^{2}+v^{2}, \quad \forall v \in K
$$


and $Q: \Omega \times K \rightarrow R^{2}$ by

$$
Q(\omega, \xi):=[-2,0] \times[-2,0]
$$

It's easy to check that $Q(\cdot, \cdot)$ is upper semicontinuous and pseudomonotone. Take $(a, b)^{\prime} \in$ $Q(\omega, \xi), \xi=\left(\xi_{1}, \xi_{2}\right)^{\prime}, \omega=\left(\omega_{1}, \omega_{2}\right)^{\prime}$ and $v=\left(v_{1}, v_{2}\right)$, then we have

$$
\left(v_{1}-\xi_{1}, v_{2}-\xi_{2}\right) \cdot(a, b)^{\prime}+\left(\omega^{2}+v^{2}\right)-\left(\omega^{2}+\xi^{2}\right) \geq 0 .
$$

Take $v_{1}=1, v_{2}=2, a=-2, b=-2$, then

$$
-\xi_{1}^{2}+2 \xi_{1}-\xi_{2}^{2}+2 \xi_{2}-1 \geq 0
$$

that is,

$$
\left(\xi_{1}-1\right)^{2}+\left(\xi_{2}-1\right)^{2}-1 \leq 0
$$

which implies that the solution set of the above stochastic variational inequality exists.

Next we prove the uniqueness of solution for GS-BMVI (1).

Theorem 3.2 Let E be a separable Banach space with a nonempty closed convex subset $K$, and let $E^{*}$ be the dual space of $E$. Suppose that a stochastic functional $\phi: \Omega \times K \rightarrow$ $(-\infty,+\infty](\phi \not \equiv+\infty)$ is convex and lower semicontinuous, while a stochastic mapping $Q$ : $\Omega \times K \rightarrow E^{*}$ is continuous and strictly monotonic about $u \in K$. If conditions (i)-(ii) and (iv)-(v) in Theorem 3.1, as well as the following condition:

$(\text { iii })^{\prime}\langle Q(\omega, u), v-u\rangle+\phi(\omega, v)$ is strictly convex and upper semicontinuous about $v \in K$, are satisfied then GS-BMVI (1) has a unique solution in $K \cap K^{\prime}$.

Proof Arguing by contradiction that $u_{1}, u_{2} \in K \cap K^{\prime}$ are two different solutions of GSBMVI (1), we have

$$
\left\langle Q\left(\omega, u_{1}\right), v-u_{1}\right\rangle+\phi(\omega, v)-\phi\left(\omega, u_{1}\right) \geq 0
$$

for $\forall v \in K$.

Taking $v=u_{2}$ in formula (7), we have

$$
\left\langle Q\left(\omega, u_{1}\right), u_{2}-u_{1}\right\rangle+\phi\left(\omega, u_{2}\right)-\phi\left(\omega, u_{1}\right) \geq 0
$$

Exchanging the position of $u_{1}$ and $u_{2}$ in formula (8) leads to

$$
\left\langle Q\left(\omega, u_{2}\right), u_{1}-u_{2}\right\rangle+\phi\left(\omega, u_{1}\right)-\phi\left(\omega, u_{2}\right) \geq 0 .
$$

Adding formulas (8) and (9), we obtain

$$
\left\langle Q\left(\omega, u_{2}\right), u_{2}-u_{1}\right\rangle-\left\langle Q\left(\omega, u_{1}\right), u_{2}-u_{1}\right\rangle \leq 0
$$


Because $Q(\omega, u)$ is strictly monotonic, we have

$$
\left\langle Q\left(\omega, u_{2}\right), u_{2}-u_{1}\right\rangle-\left\langle Q\left(\omega, u_{1}\right), u_{2}-u_{1}\right\rangle=0
$$

For any $u \in K,\langle Q(\omega, u), v-u\rangle+\phi(\omega, v)$ is strictly convex, so we let $v=\frac{1}{2}\left(u_{1}+u_{2}\right)$ in formula (7), and then

$$
\begin{gathered}
\frac{1}{2}\left[\phi\left(\omega, u_{1}\right)+\phi\left(\omega, u_{2}\right)\right]+\frac{1}{2}\left[\left\langle Q\left(\omega, u_{1}\right), u_{1}-u_{1}\right\rangle+\left\langle Q\left(\omega, u_{1}\right), u_{2}-u_{1}\right\rangle\right] \\
>\phi\left(\omega, \frac{1}{2}\left(u_{1}+u_{2}\right)\right)+\left\langle Q\left(\omega, u_{1}, \frac{1}{2}\left(u_{2}-u_{1}\right)\right)\right\rangle \geq \phi\left(\omega, u_{1}\right),
\end{gathered}
$$

so

$$
\left\langle Q\left(\omega, u_{1}\right), u_{1}-u_{1}\right\rangle+\left\langle Q\left(\omega, u_{1}\right), u_{2}-u_{1}\right\rangle>\phi\left(\omega, u_{1}\right)-\phi\left(\omega, u_{2}\right) .
$$

Because $\left\langle Q\left(\omega, u_{1}\right), u_{1}-u_{1}\right\rangle=0$, one has

$$
\left\langle Q\left(\omega, u_{1}\right), u_{2}-u_{1}\right\rangle>\phi\left(\omega, u_{1}\right)-\phi\left(\omega, u_{2}\right) .
$$

Exchanging the position of $u_{1}$ and $u_{2}$ of formula (10) gives

$$
\left\langle Q\left(\omega, u_{2}\right), u_{1}-u_{2}\right\rangle>\phi\left(\omega, u_{2}\right)-\phi\left(\omega, u_{1}\right) .
$$

Adding formulas (10) and (11) results in

$$
\left\langle Q\left(\omega, u_{1}\right), u_{2}-u_{1}\right\rangle+\left\langle Q\left(\omega, u_{2}\right), u_{1}-u_{2}\right\rangle>0,
$$

that is,

$$
\left\langle Q\left(\omega, u_{2}\right), u_{2}-u_{1}\right\rangle+\left\langle Q\left(\omega, u_{1}\right), u_{2}-u_{1}\right\rangle<0,
$$

which yields a contradiction. Therefore, the uniqueness of solution for GS-BMVI (1) is proved.

Remark 3.2 The convexity is strengthened in condition (iii) of Theorem 3.2 based on Theorem 3.1, and we strengthen the monotonicity condition of the stochastic mapping $Q$. It is essential to discuss the monotonicity and convexity when we analyze the uniqueness of solutions for variational inequalities.

Example 3.2 Let $E=R^{2}$ and $K=[0,+\infty) \times[0,+\infty)$. Suppose that $\omega$ has a normal distribution. Define $\phi: \Omega \times K \rightarrow(-\infty,+\infty]$ by

$$
\phi(\omega, v):=\omega^{2}+v^{2}, \quad \forall v \in K
$$

and $Q: \Omega \times K \rightarrow R^{2}$ by

$$
Q(\omega, \xi):=2 \omega+\xi
$$


It's easy to check that $Q(\cdot, \cdot)$ is upper semicontinuous and monotone. Taking $\xi=\left(\xi_{1}, \xi_{2}\right)^{\prime}$, $\omega=\left(\omega_{1}, \omega_{2}\right)^{\prime}$ and $v=\left(v_{1}, v_{2}\right)$, we have

$$
\left(v_{1}-\xi_{1}, v_{2}-\xi_{2}\right)\left(\begin{array}{l}
2 \omega_{1}+\xi_{1} \\
2 \omega_{2}+\xi_{2}
\end{array}\right)+\left(\omega^{2}+v^{2}\right)-\left(\omega^{2}+\xi^{2}\right) \geq 0 .
$$

Taking $v_{1}=1, v_{2}=2$ and $v_{1}=2, v_{2}=1$, respectively, we have

$$
\left\{\begin{array}{l}
-2 \xi_{1}^{2}+\left(1-2 \omega_{1}\right) \xi_{1}-2 \xi_{2}^{2}+\left(2-2 \omega_{2}\right) \xi_{2}+2 \omega_{1}+4 \omega_{2}+5 \geq 0 \\
-2 \xi_{1}^{2}+\left(2-2 \omega_{1}\right) \xi_{1}-2 \xi_{2}^{2}+\left(1-2 \omega_{2}\right) \xi_{2}+4 \omega_{1}+2 \omega_{2}+5 \geq 0
\end{array}\right.
$$

which implies that there exists a unique solution for the above stochastic variational inequality.

We now discuss the compactness and convexity of the solution set for GS-BMVI (1).

Theorem 3.3 Let E be a separable Banach space with a nonempty closed and convex subset, and let $E^{*}$ be the dual space of $E$. Suppose that a stochastic functional $\phi: \Omega \times K \rightarrow$ $(-\infty,+\infty](\not \equiv+\infty)$ is convex, while a stochastic mapping $Q: \Omega \times K \rightarrow E^{*}$ is monotone and continuous. If conditions (i)-(ii) and (iv)-(v) in Theorem 3.1, as well as the following conditions:

$(\text { iii })^{\prime}\langle Q(\omega, u), v-u\rangle+\phi(\omega, v)$ is convex and upper semicontinuous about $v \in K$;

(vi) $\langle Q(\omega, u), v-u\rangle$ and $\phi(\omega, v)$ are lower semicontinuous about $v \in K$, are satisfied, then the solution set of GS-BMVI (1) is compact and convex in $K \cap K^{\prime}$.

Proof Before the proof, Minty's lemma [21] is introduced as follows:

Let $E$ be a Hausdorff topological vector space with a closed convex subset $K$. Suppose that the stochastic functional $\phi$ and the stochastic mapping $Q$ satisfy the following conditions:

(i) $\phi: \Omega \times K \rightarrow(-\infty,+\infty]$ with $\phi \not \equiv+\infty$ is lower semicontinuous about $v \in K$.

(ii) $Q: \Omega \times K \rightarrow E^{*}$ is monotone, semicontinuous, and lower semicontinuous about $v \in K$.

If for any $u \in K,\langle Q(\omega, u), v-u\rangle+\phi(\omega, v)$ is convex about $v$, then there exists $\bar{u} \in K$ such that $G(\omega, v)=\{\bar{u} \in K:\langle Q(\omega, \bar{u}), v-\bar{u}\rangle+\phi(\omega, v)-\phi(\omega, \bar{u}) \geq 0\}$ and $H(\omega, v)=\{u \in K:$ $\langle Q(\omega, v), \bar{u}-v\rangle+\phi(\omega, \bar{u})-\phi(\omega, v) \leq 0\}$ coincide.

Based on this, we will then focus on the compactness and convexity of the solution set. Let $S$ be the solution set of GS-BMVI (1) in $K \cap K^{\prime}$, so

$$
\bar{u} \in S=\bigcap_{v \in K} G(\omega, v) \neq \varnothing .
$$

For each $v \in K$, let

$$
H(\omega, v)=\{\bar{u} \in K:\langle Q(\omega, v), \bar{u}-v\rangle+\phi(\omega, \bar{u})-\phi(\omega, v) \leq 0\}
$$

then, by Minty's lemma, we have $\bigcap_{v \in K} G(\omega, v)=\bigcap_{v \in K} H(\omega, v)$. 
From conditions (iii) and (vi) in Theorem 3.3, we know that $\langle Q(\omega, v), \bar{u}-v\rangle+\phi(\omega, \bar{u})$ is convex and lower semicontinuous, so $H(\omega, v)$ is closed and convex, then $S=\bigcap_{v \in K} H(\omega, v)$ is also closed convex in $E$, thus,

$$
\bigcap_{v \in K} H(\omega, v)=\overline{\bigcap_{v \in K} G(\omega, v)} \subset \overline{G(\omega, k)} .
$$

Arguing by contradiction, suppose that there exists $u \in G(\omega, k)$ and $u \notin K^{\prime}$, so $u \in K \backslash K^{\prime}$, then from condition (ii) in Theorem 3.1,

$$
\langle Q(\omega, u), k-u\rangle+\phi(\omega, k)-\phi(\omega, u)<0
$$

which is a contradiction with $u \in G(\omega, k)$, so $G(\omega, k) \subset K^{\prime}$.

Because $K^{\prime}$ is a compact subset, $K^{\prime}$ is closed, and then from (13) we get that

$$
\overline{G(\omega, k)} \subset K^{\prime}
$$

Therefore, $S$ is a compact convex set in $K$, which means that $S$ is compact and convex in $K \cap K^{\prime}$.

Remark 3.3 Minty's technique [21] is applied in the proof, and it is also utilized for the stochastic variational inequalities in Zhou [22] and Lee and Salahuddin [23]. Besides, the above study on the compactness and convexity of the solution set for GS-BMVI (1) can help us deal with general infinite-dimensional problems as with the finite-dimensional problems we considered.

\section{Conclusion}

In this paper we introduced a class of generalized stochastic Browder mixed variational inequalities. First, the existence of a solution set for GS-BMVI (1) in separable Banach space $E$ with a closed convex subset $K$ was studied based on FKKM theorem and Aumman's measurable selection theorem. Next, the existence of solution sets in a separable Banach space $E$ with a compact convex subset $K$ and in a separable reflexive Banach space $E$ with a closed convex subset $K$ were presented. Then the uniqueness of the solution for GS-BMVI (1) was proved, which implies that the property of uniqueness is related to the strict convexity of the mapping $\phi$ and the strict monotonicity of the mapping $Q$. In addition, the compactness and convexity were studied through the Minty's technique to help us deal with general infinite-dimensional problems.

The work of this paper is a generalization of Browder mixed variational inequalities in the stochastic situation. Compared with [24], we extended the environment of stochastic variational inequalities to Banach spaces, and obtained the existence of solutions of GSBMVI (1) in a separable Banach space and in a reflexive Banach space. The results showed that the existence of solutions can be obtained in a reflexive Banach space by properly weakening the conditions on the subset $K$. Besides, we not only studied the existence and uniqueness of solutions, but also discussed the compactness and the convexity of such solution sets. Compared with [19], we facilitated further research on a class of stochastic evolutionary problems driven by stochastic variational inequalities inspired by [25]. 
Further research could focus on the following situation: let $E_{1}$ and $E_{2}$ be two separable Banach spaces, $K$ be a nonempty convex subset of $E_{1}, E_{1}^{*}$ be the dual space of $E_{1}, L\left(E_{1}, E_{2}\right)$ be the space of linear operators from $E_{1}$ to $E_{2}$. For a measurable space $(\Omega, \mathcal{F})$, we set a stochastic evolutionary problem as follows:

$$
\left\{\begin{array}{l}
d x(t)=A x(t) d t+\mu(t, x(t)) d t+\sigma\left(\omega, t, x(t), u_{\omega}(t)\right) d B(t), \quad \omega \in \Omega, t \in[0, T] \\
u_{\omega}(t) \in S(K, g(\omega, t, x(t), \cdot), \phi), \quad \omega \in \Omega, t \in[0, T] \\
x(0)=x_{0} .
\end{array}\right.
$$

where the operator $A$ is the infinitesimal generator of a bounded linear operator; $\mu$ : $\Omega \times[0, T] \times E_{2} \rightarrow E_{2}$ and $\sigma: \Omega \times[0, T] \times E_{2} \times K \rightarrow 2^{L\left(E_{1}, E_{2}\right)}$ are given functions; $B$ is Brownian motion; $S(K, g(\omega, t, x(t), \cdot), \phi)$ stands for the solution set of the stochastic variational inequality, where we want to find $u: \Omega \times[0, T] \rightarrow K$ such that

$$
\left\langle g\left(\omega, t, x(t), u_{\omega}(t)\right), v-u_{\omega}(t)\right\rangle+\phi(\omega, v)-\phi\left(\omega, u_{\omega}(t)\right) \geq 0, \quad \forall v \in K
$$

Here, $g: \Omega \times[0, T] \times E_{2} \times K \rightarrow E_{1}^{*}$ is a stochastic mapping; $\phi: \Omega \times E_{1} \rightarrow(-\infty,+\infty]$, satisfying $\phi \neq+\infty$, is a stochastic functional. To obtain the solution of problem (*), the key, as indicated in [25], is to guarantee that some properties of the solution sets $S(K, g(\omega, t, x(t), \cdot), \phi)$ hold for the stochastic variational inequality $\left.{ }^{* *}\right)$, such as convexity and continuity, which will be discussed in our further work.

\section{Acknowledgements}

The authors are grateful to the editor and the reviewers, whose valuable comments and suggestions have led to a considerable improvement of this paper. The authors would like to thank the financial support of the grants for overseas returnees from Sichuan provincial human resources and social security department.

Funding

This work was supported by the National Natural Science Foundation of China (11601451, 11526173).

\section{Availability of data and materials}

The data involved in the examples of our manuscript are included within the article.

\section{Competing interests}

The authors declare that they have no competing interests.

\section{Authors' contributions}

Conceptualization, CM; Writing of original draft, CM; Methodology, CM; Writing of review and editing, FF; Proof of Theorem 3.1, FF; Validation, ZY and XL. All authors read and approved the final manuscript.

\section{Author details}

${ }^{1}$ School of Science, Southwest Petroleum University, Chengdu, P.R. China. ${ }^{2}$ Institute for Artificial Intelligence, Southwest Petroleum University, Chengdu, P.R. China. ${ }^{3}$ State Key Laboratory of Oil and Gas Reservoir Geology and Exploitation, Southwest Petroleum University, Chengdu, P.R. China.

\section{Publisher's Note}

Springer Nature remains neutral with regard to jurisdictional claims in published maps and institutional affiliations.

Received: 5 July 2019 Accepted: 29 December 2019 Published online: 09 January 2020

\section{References}

1. Li, X., Zou, Y.Z.: Existence result and error bounds for a new class of inverse mixed quasi-variational inequalities. J. Inequal. Appl. 2016, 42, 1-13 (2016)

2. Tang, Y.X., Guan, J.Y., Xu, Y.C., et al.: A kind of system of multivariate variational inequalities and the existence theorem of solutions. J. Inequal. Appl. 2017, 208 (2017)

3. Tang, G.J., Li, Y.S.: Uniqueness and stability of the solution for strongly pseudomonotone variational inequalities in Banach spaces. Adv. Math. 47(3), 137-144 (2018) (in Chinese) 
4. Díaz, J..., Véron, L.: Existence theory and qualitative properties of the solutions of some first order quasilinear variational inequalities. Indiana Univ. Math. J. 32(3), 319-361 (2020)

5. Daniele, P., Giuffré, S.: Random variational inequalities and the random traffic equilibrium problem. J. Optim. Theory Appl. 167(1), 1-19 (2014)

6. Jadamba, B., Raciti, F.: Variational inequality approach to stochastic Nash equilibrium problems with an application to Cournot oligopoly. J. Optim. Theory Appl. 165(3), 1050-1070 (2015)

7. Bensoussan, A., Li, Y., Yam, S.C.P.: Backward stochastic dynamics with a subdifferential operator and non-local parabolic variational inequalities. Stoch. Process. Appl. 128(2), 644-688 (2017)

8. Barbagallo, A., Scilla, G.: Stochastic weighted variational inequalities in non-pivot Hilbert spaces with applications to a transportation model. J. Math. Anal. Appl. 457(2), 1118-1134 (2017)

9. Chen, X.J., Pong, T.K., Wets, J.B.: Two-stage stochastic variational inequalities: an ERM-solution procedure. Math. Program. 165(1), 71-111 (2017)

10. Rockafellar, R.T., Sun, J.: Solving monotone stochastic variational inequalities and complementarity problems by progressive hedging. Math. Program. (2018). https://doi.org/10.1007/s10107-018-1251-y

11. Ren, J.G., Shi, Q., Wu, J.: Limit theorems for stochastic variational inequalities with non-Lipschitz coefficients. Potential Anal. (2018). https://doi.org/10.1007/s11118-018-9704-8

12. Zhang, S.S., Huang, N.J.: Random generalized set-valued quasi-complementarity problems. Acta Math. Appl. Sin. 16(3), 396-405 (1993)

13. Ravat, U., Shanbhag, U.V.: On the existence of solutions to stochastic quasi-variational inequality and complementarity problems. Math. Program. 165(1), 291-330 (2017)

14. Zhang, S.S., Ma, Y.H.: KKM technique and its applications. Appl. Math. Mech. 14(1), 11-20 (1993)

15. Fan, K.: A generalization of Tychonoff's fixed point theorem. Math. Ann. 142(3), 305-310 (1961)

16. Tan, K.K., Yuan, X.Z.: On deterministic and random fixed points. Proc. Am. Math. Soc. 119(3), 849-856 (1993)

17. Aumann, R.J. Integrals of set-valued functions. J. Math. Anal. Appl. 12(1), 1-12 (1965)

18. Himmelberg, C.J., Parthasarathy, T., Vleck, F.S.V.: On measurable relations. Fundam. Math. 111(2), 52-72 (1981)

19. Chuong, N.M., Thuan, N.X.: Random nonlinear variational inequalities for mappings of monotone type in Banach spaces. Stoch. Anal. Appl. 24(3), 489-499 (2006)

20. Wu, D.P.: On random variational inequalities and random complementarity problem. Sichuan Daxue Xuebao 28(5), 535-537 (2005)

21. Minty, G.J.: On the generalization of a direct method of the calculus of variations. Bull. Am. Math. Soc. 73(3), 315-322 (1967)

22. Zhou, W: A class of generalized random nonlinear variational inequalities in Hilbert spaces. J. Southwest Univ. Natur. Sci. Ed. 42(4), 443-445 (2016)

23. Lee, B.S., Salahuddin: Minty lemma for inverted vector variational inequalities. Optimization 66(3), 351-359 (2017)

24. Han, Y., Huang, N.J., Lu, J.: Existence and stability of solutions to inverse variational inequality problems. Appl. Math. Mech. 38(5), 137-152 (2017)

25. Liu, Z.H., Zeng, S., Motreanu, D.: Evolutionary problems driven by variational inequalities. J. Differ. Equ. 260(9), 6787-6799 (2016)

\section{Submit your manuscript to a SpringerOpen ${ }^{\circ}$ journal and benefit from:}

- Convenient online submission

- Rigorous peer review

- Open access: articles freely available online

- High visibility within the field

- Retaining the copyright to your article

Submit your next manuscript at $\gg$ springeropen.com 